\title{
Biological specific recognition of glycopolymer- modified interfaces by RAFT living radical polymerization
}

\author{
Masayuki Toyoshima, Tomoyuki Oura, Tomohiro Fukuda, Erino Matsumoto and Yoshiko Miura
}

Glycopolymers with $\alpha$-galactose ( $\alpha$-Gal) and $\alpha$-mannose ( $\alpha$-Man) were synthesized by means of living radical polymerization with a reversible addition-fragment chain transfer reagent, and the thin-layer formation of glycopolymers was investigated in terms of protein recognition abilities. Thiol-terminated glycopolymers formed a thin layer of about $2.5 \mathrm{~nm}$ in thickness on a gold substrate, and the glycopolymer thin layer showed specific interaction with sugar recognition proteins (lectins and Shiga toxins (Stxs)). The interactions were highly specific, and the signal-to-noise ratio of protein recognition was greater than 16 . Glycopolymer-substituted gold nanoparticles (GNPs) also showed biorecognition abilities and protein-specific aggregation. The protein recognition abilities of the GNPs were also analyzed. The glycopolymer-substituted GNPs were utilized for signal amplification of surface plasmon resonance (SPR) to detect protein-saccharide recognition. The glycopolymer with $\alpha$-Gal showed a strong interaction with Stxs according to SPR measurements, suggesting a possible application of $\alpha$-Gal-substituted GNPs in Stx-1 biosensing. Polymer Journal (2010) 42, 172-178; doi:10.1038/pj.2009.321; published online 23 December 2009

Keywords: biointerface; glycopolymer; RAFT living radical polymerization; saccharide

\section{INTRODUCTION}

Saccharides on cell surfaces have important roles in life systems, such as cell-cell communication, immune response, pathogen invasion and cancer metastasis. ${ }^{1}$ Because saccharide-protein interactions regulate life phenomena, a better understanding of saccharide functions would greatly aid elucidation of such phenomena and, hence, the diagnosis and detection of disease and pathogens. ${ }^{2}$

Saccharide-protein interactions are generally too weak to be used in biomaterials and devices, but interestingly, the interactions can be amplified by multivalency. Multivalent compounds of glycopolymers have been reported to exhibit strong saccharide-protein interactions. ${ }^{3-5}$ We prepared various glycopolymers that can be used for cell cultivation, ${ }^{6}$ toxin neutralization ${ }^{7}$ and $A \beta$-inhibition. ${ }^{8}$ In addition, saccharide-protein interactions are difficult to detect because of their lack of fluorescence or conductivity. Thus, conjugate materials with nanoparticles or substrates provide suitable materials for biosensing. We previously reported the preparation of glycopolymer-substituted gold nanoparticles (GNPs) and demonstrated the detection of proteinsaccharide interactions by colorimetric changes. ${ }^{9}$

In this study, we investigated glycopolymer-substituted substrates and nanoparticles. We prepared glycopolymer-substituted substrates by reversible addition-fragment chain transfer (RAFT) living radical polymerization, ${ }^{9-11}$ in which the terminal group of dithioester was converted to thiol to form a polymer layer through Au-S bonding on GNPs ${ }^{12,13}$ and substrates by the 'grafting to' method. ${ }^{14}$ Glycopolymers of polyacrylamide derivatives with $\alpha$-galactose $(\alpha$-Gal) and $\alpha$-mannose $(\alpha-\mathrm{Man})$ and the corresponding thin layers were prepared. Precise control of the polymer resulted in a uniform biological interface, which led to a sensitive biosensing substrate.

The gold substrates and nanoparticles can be applied to various sensing methods such as surface plasmon resonance (SPR), quartz crystal microbalance, electrochemistry and colorimetric changes in nanoparticles. We measured the affinities and specificities of the glycopolymer thin layer to proteins, using SPR for the gold substrates and using colorimetric changes for the GNPs. In addition to the model interactions between lectin and saccharide, practical biosensing for Shiga toxins (Stxs) was also studied in this investigation using an $\alpha$-Gal-substituted glycopolymer. ${ }^{15}$

\section{EXPERIMENTAL PROCEDURE}

Materials

The following reagents were used as received: D-mannose (Man) (Kishida Chemical, Osaka, Japan), $p$-nitrophenyl $\alpha$-D-galactoside, acryloyl chloride (TCI, Tokyo, Japan), p-nitrophenol, 2.2'-azobis(2-amidinopropane)dihydrochloride (Kanto Chemical, Tokyo, Japan), bovine serum albumin (BSA), fibrinogen, lysozyme, (thiobenzoyl)thioglycolic acid (Sigma-Aldrich, Louisiana, MO, USA), peanut agglutinin (PNA), concanavalin A (ConA) (J-oil Mills, Tokyo, Japan), Stx-1 (Vero toxin-1 (Stx-1, from Escherichia coli O-157)) (Nacalai Tesque, Kyoto, Japan), high-flow nitrocellulose membrane (FH135) absorption pads (Millipore, Billerica, MA, USA), GNPs $(40 \mathrm{~nm}$ ) (Tanaka Kikinzoku, Tokyo, Japan) and a silver enhancer kit for membrane application (Funakoshi, Tokyo, Japan). 
Acrylamide (AAm) (Kanto Chemical) was used after recrystallization from chloroform and methanol. Acetylene-terminated disulfide and azide-terminated $\alpha$-Man were synthesized according to the literature. ${ }^{16,17}$

\section{Characterization}

${ }^{1} \mathrm{H}(300 \mathrm{MHz})$ and ${ }^{13} \mathrm{C}(75 \mathrm{MHz})$ nuclear magnetic resonance spectra were recorded in $\mathrm{CDCl}_{3}$ and $\mathrm{D}_{2} \mathrm{O}$ using a Varian Gemini 300 spectrometer (Varian, Palo Alto, CA, USA) equipped with a Sun workstation. Gel permeation chromatography was carried out with a JASCO 800 high-performance liquid chromatographer (JASCO, Tokyo, Japan) with Shodex SB803 HQ columns (Showa Denko K. K., Tokyo, Japan) and phosphate-buffered saline as the eluent. Molecular weights were evaluated relative to a pullulan standard. The contact angle was determined using a Drop Master 300 (Kyowa Interface Science, Saitama, Japan). Infrared spectra were obtained at room temperature using an FTIR Spectrum 100 (PerkinElmer, Waltham, MA, USA). Fourier transform infrared-reflection absorption spectroscopy was carried out using a Reflector 2 instrument (Harrick Scientific, Pleasantville, NY, USA). The bonding on the gold substrates was measured by quartz crystal microbalance using a Q-SENSE D300 (QSENSE, Frölunda, Sweden) with a gold substrate of QSX301. SPR spectra were recorded using a BIACORE 1000 (GE Healthcare UK Ltd, Little Chalfont, UK) with phosphate-buffered saline. Atomic force microscopy images were recorded with a VN-8000 instrument equipped with an op-75042 cantilever (Keyence, Kyoto, Japan). Transmission electron microscopy images were recorded at $100 \mathrm{kV}$ using a Hitachi HF-2000 (Hitachi, Tokyo, Japan) instrument equipped with an AMT-CCD camera (Hitachi). Gold nanoparticles (GNPs) for transmission electron microscopy were prepared by the deposition of buffer-dispersed particles onto a carbon film supported by a copper grid. Immunochromatography was conducted with high-flow nitrocellulose membrane (FH135) absorption pads (Millipore).

\section{General procedure for polymerization}

$p$-(N-Acrylamidophenyl)pyranosides (1-1 and 1-2) were synthesized according to previous procedures (Scheme 1). ${ }^{18} \mathrm{~A}$ mixture of the corresponding sugar monomer, AAm, an initiator (2.2'-azobis(2-amidinopropane)dihydrochloride),
RAFT reagent and solvent in a Pyrex tube was degassed by three freeze-thaw cycles. The tube was sealed under vacuum and heated at $60^{\circ} \mathrm{C}$ with occasional agitation. Polymerization was terminated by cooling the tube, and the product was precipitated in acetone and collected by centrifugal sedimentation. Polymers were hydrogenated with $\mathrm{NaBH}_{4}$ to obtain a thiol terminal. Further details are given in Supplementary information.

\section{Preparation of glycopolymer-substituted gold} substrates and nanoparticles

Gold substrates from GE Healthcare (SPR sensor chip Au) were used for SPR measurements, and gold substrates from Moritex Co. (Tokyo, Japan) were used for infrared, X-ray photoelectron spectroscopy and ellipsometry measurements. ${ }^{9}$ The substrates were cleaned by ultraviolet/ozone irradiation (Bioforce Nanosciences, Ames, IA, USA) for $30 \mathrm{~min}$ before use. The thiol-terminated polymer was dissolved in MilliQ water at a concentration of $1.0 \mathrm{mg} \mathrm{ml}^{-1}$. The substrate was immersed in the solution for $24 \mathrm{~h}$, then rinsed with MilliQ water and dried under $\mathrm{N}_{2}$.

Glycopolymer-substituted GNPs were prepared according to our previous report. Glycopolymers were mixed with an aqueous dispersion of GNPs for $12 \mathrm{~h}$, and the resulting glycopolymer-substituted GNPs were purified by centrifugation $(540000 \mathrm{~g}, 2 \mathrm{~h})$

\section{SPR measurements}

Saccharide-protein interactions were analyzed according to SPR measurements obtained with a BIACORE 1000 (GE Healthcare UK Ltd) on glycopolymerimmobilized gold substrates. The flow rate of the buffer was $30 \mu \mathrm{min}^{-1}$, and the sample solution was injected for $20 \mathrm{~s}$. The substrate was regenerated twice by treatment with $10 \mathrm{~mm}$ glycine at $\mathrm{pH} 1.5$ (GE Healthcare Bioscience, Little Chalfont, UK) as the dissociate solution for $2 \mathrm{~min}$. Lectin binding to the corresponding saccharides (ConA to $\alpha$-Man and PNA to Gal) was calculated from sensorgrams with the assumption of Langmuir binding (1:1) using BIAevaluation software (GE Healthcare UK Ltd). In the case of Stx-1, binding was calculated from Scatchard plots.

In the case of SPR signal amplification with GNPs, the flow rate of the buffer was $10 \mu \mathrm{min}^{-1}$; the sample solution was injected for $60 \mathrm{~s}$ and then<smiles>[R]NC(=O)C(C)CC(C)C(C)CC(C)(CC(=O)O)CC(C)C(=O)N[R]</smiles>

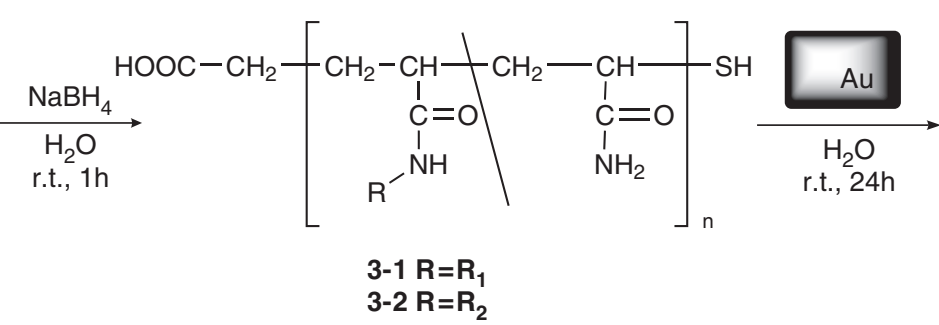<smiles>[R]NC(=O)C(CC(C)CC(=O)O)CC(CC)C(N)=O</smiles>
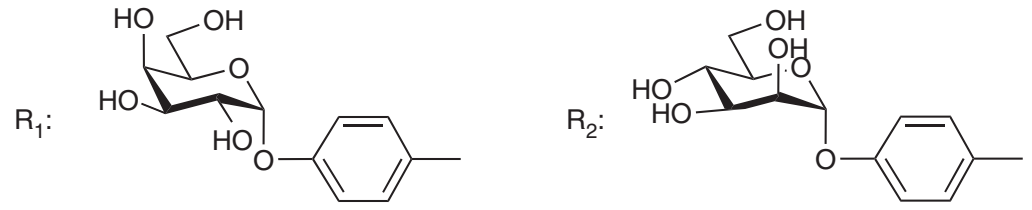

$R_{3}: \quad H$ 
glycopolymer-substituted GNPs $\left(\mathrm{OD}_{529}=0.45\right)$ were injected for $60 \mathrm{~s}$. The substrate was regenerated twice by treatment with $10 \mathrm{~mm}$ glycine at $\mathrm{pH} 1.5$ (GE Healthcare Bioscience) as the dissociate solution for $5 \mathrm{~min}$. Binding with PNA (a Gal-binding protein) and the nonspecific adsorption of fibrinogen were analyzed.

\section{Immunochromatography}

A solution of Stx-1 $(2.4 \mu \mathrm{M}, 10 \mu \mathrm{l})$ was applied to a nitrocellulose membrane as a test spot. ${ }^{19}$ After drying for $30 \mathrm{~min}$ at room temperature, the membrane was blocked against nonspecific protein adsorption by immersion in $50 \mathrm{~mm}$ boric acid buffer containing $0.5 \%$ casein ( $\mathrm{pH} 8.5$ ) and incubated for $30 \mathrm{~min}$ at room temperature. The blocked membrane was then washed for $30 \mathrm{~min}$ at room temperature by immersion in $5.0 \mathrm{~mm}$ phosphate buffer $(\mathrm{pH} 7.5)$ containing $0.01 \%$ sodium dodecyl sulfate. After drying for $3 \mathrm{~h}$, the membrane was prepared on a backing sheet, and an absorbent pad was attached. The solution of glycopolymer-substituted GNPs $\left(\mathrm{OD}_{529}=4.5\right)$ was placed on the membrane for $30 \mathrm{~min}$ and washed with MilliQ water $(18.3 \mathrm{M} \Omega-\mathrm{cm})$. The membrane was incubated with the prepared silver enhancer $(200 \mu \mathrm{l}$ each of solutions A and B) for $30 \mathrm{~min}$. After a suitable color intensity was attained, the membrane was rinsed for $3 \mathrm{~min}$ in a continuous stream of MilliQ water.

\section{RESULTS AND DISCUSSION}

\section{Polymerization of glycopolymer}

Polymerization of 1-1 and 1-2 (Scheme 1) was conducted with the RAFT reagent (thiobenzoyl)thioglycolic acid in a mixture of water and DMSO; polymers were obtained with low dispersities $(<1.5)$ (Table 1). The yields of polymers increased with polymerization time, but the yield did not exceed $30 \%$ after $24 \mathrm{~h}$. The yield of the polymer was low because of amine impurities from AAm, which induce degradation of the RAFT reagent to amine. ${ }^{20}$ Amine was generated because of the aqueous solvent; thus, we are investigating RAFT living radical polymerization of glycopolymers with more appropriate conditions and RAFT reagents.

\section{Preparation of glycopolymer on the gold substrate}

The glycopolymers of Run no. 3 (P3) and 6 (P6) were hydrogenated to result in a terminal thiol group, and the gold substrates were modified by the addition of $1 \mathrm{wt} \%$ glycopolymer solution, which we called P3gold and P6-gold, respectively. The glycopolymers used were selected by following the previous report. ${ }^{9}$ The contact angle of the substrate obtained was rapidly reduced by the addition of glycopolymers, from $82^{\circ}$ to $24^{\circ}$ after $0.5 \mathrm{~h}$, and stabilized at $14.5^{\circ}$ after $12 \mathrm{~h} .{ }^{21}$ Formation of the polymer layer was also confirmed by Fourier transform infraredreflection absorption spectroscopy; bands for the hydroxyl groups ( $\delta \mathrm{OH}$ about $\left.3300 \mathrm{~cm}^{-1}\right)$, amide $\mathrm{I}\left(\mathrm{VC}=\mathrm{O}\right.$ at $\left.1680 \mathrm{~cm}^{-1}\right)$ and amide II $\left(\delta \mathrm{NH}\right.$ at $\left.1518 \mathrm{~cm}^{-1}\right)$ (Figure 1$)$, were observed. The thicknesses of P3- and P6-gold were measured using ellipsometry and atomic force microscopy as $2.5 \pm 0.2 \mathrm{~nm}$ (Supplementary information). The area per molecule of the glycopolymer as determined by quartz crystal microbalance was $1.6 \mathrm{~nm}^{2}$, following the Sauerbrey equation in air (Supplementary Information). These values for thickness and bonding indicate a polymer layer with random orientation and a non-dense structure, indicating that a pancake layer formation occurred because of the 'grafting to' method. ${ }^{22}$

\section{SPR analyses of glycopolymer-protein interactions}

Substrates with glycopolymers were subjected to interaction evaluation with proteins (Con A, PNA, BSA, lysozyme and fibrinogen) by SPR measurements. The time course of the resonance unit (RU) change was monitored for the glycopolymer-substituted substrates: a representative time-course curve of the RU with a 500-nM protein injection is shown in Figure 2. The glycopolymer-substituted substrate showed strong interaction with the corresponding lectins because of the multivalent effect of the glycopolymer. ${ }^{3}$ The $K_{\mathrm{A}}$ values of P3-gold to PNA and P6-gold to ConA were $2.0 \times 10^{7}$ and $1.6 \times 10^{7}\left(\mathrm{M}^{-1}\right)$, respectively (Table 2 ).

Protein adsorption was compared in terms of specific recognition (Figure 2 and Table 3). The $\mathrm{RU}_{\mathrm{eq}}$ change of P3-gold for PNA (500 nM) was $8.1 \times 10^{2}$, whereas the change for other proteins was at the most $31(\mathrm{~S} / \mathrm{N}>26)$. The $\mathrm{RU}_{\text {eq }}$ change of P6-gold with ConA was $2.4 \times 10^{3}$, and the change for those with noncorresponding proteins were at the most $1.5 \times 10^{2}(\mathrm{~S} / \mathrm{N}>16)$. These SPR results indicate the formation of a highly bio-specific poly(sugar-co-AAm) surface because of the hydrophilic polymer layer. We also prepared an $\alpha$-Gal monolayer without a polymer backbone and measured the interaction with proteins by SPR (Figure 3 ). The $\alpha$-Gal monolayer showed an interaction with the corresponding lectin of PNA, but the specificity was less than that for glycopolymer substrates. For example, the amount of fibrinogen bound to the substrate was

Table 1 Polymerization of $p$-acrylamidophenyl $\alpha$-Gal (1-1) and $p$-acrylamidophenyl $\alpha$-Man (1-2) with acrylamide (1-3) in the presence of RAFT reagent ${ }^{\mathrm{a}}$

\begin{tabular}{|c|c|c|c|c|c|c|c|}
\hline \multirow[b]{2}{*}{ Run no. } & \multicolumn{2}{|c|}{ Monomer } & \multirow[b]{2}{*}{ Time $(h)$} & \multirow[b]{2}{*}{ Yield (\%) } & \multirow[b]{2}{*}{$10^{-3} \mathrm{Mn}\left(\mathrm{g} \mathrm{mol}^{-1}\right)^{\mathrm{b}}$} & \multirow[b]{2}{*}{$M w / M n$} & \multirow[b]{2}{*}{ Sugar ratio in polymer ${ }^{c}$} \\
\hline & Saccharide monomer (mmol) & Acrylamide (mmol) & & & & & \\
\hline \multirow[t]{2}{*}{1} & $1-1$ & $1-3$ & 2 & 7.7 & 5.3 & 1.2 & 0.53 \\
\hline & 0.030 & 0.27 & & & & & \\
\hline \multirow[t]{2}{*}{2} & $1-1$ & $1-3$ & 3 & 13 & 6.6 & 1.3 & 0.38 \\
\hline & 0.030 & 0.27 & & & & & \\
\hline \multirow[t]{2}{*}{3} & $1-1$ & $1-3$ & 4 & 17 & 7.0 & 1.4 & 0.24 \\
\hline & 0.030 & 0.27 & & & & & \\
\hline \multirow[t]{2}{*}{4} & $1-1$ & $1-3$ & 5 & 19 & 10 & 1.4 & 0.27 \\
\hline & 0.030 & 0.27 & & & & & \\
\hline \multirow[t]{2}{*}{5} & $1-1$ & $1-3$ & 24 & 34 & 12 & 1.4 & 0.20 \\
\hline & 0.030 & 0.27 & & & & & \\
\hline \multirow[t]{2}{*}{6} & $1-2$ & $1-3$ & 4 & 20 & 8.1 & 1.3 & 0.25 \\
\hline & 0.030 & 0.27 & & & & & \\
\hline
\end{tabular}

aMonomer $0.30 \mathrm{mmol}$, AAPD $0.20 \mathrm{~mol} \%$, (thiobenzoyl)thioglycolic acid $0.50 \mathrm{~mol} \%, 60^{\circ} \mathrm{C}$

bBy pullulan standard.

CDetermined using $300 \mathrm{MHz}{ }^{1} \mathrm{H}$ NMR. 

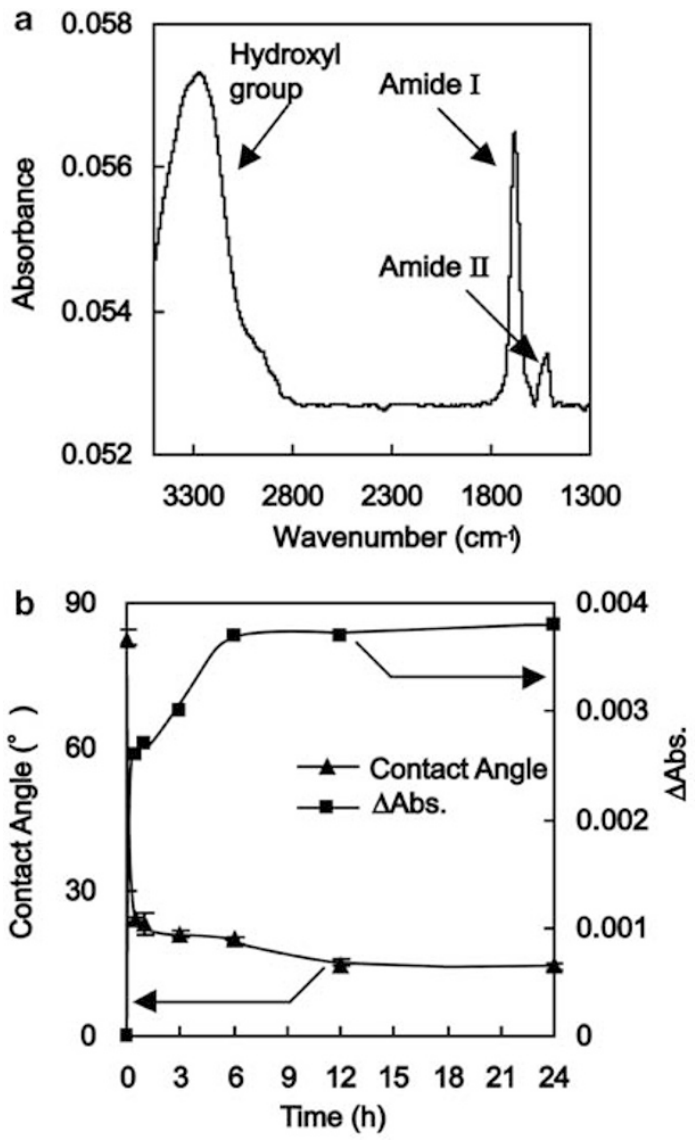

Figure 1 The characterization of a thin layer of glycopolymer (P3). (a) Fourier transform infrared-reflection absorption spectroscopy spectrum of P3-gold. (b) Time-course changes of the contact angle and the absorbance of amide $\mathrm{I}$.

more than that of PNA, and the $\mathrm{RU}_{\max }$ values of ConA, lysozyme and BSA were only $40 \%$ of that of PNA. In addition, the amount of PNA bound to the monolayer was only $20 \%$ of that of glycopolymer P3-gold.

Basically, the sugar-immobilized substrates show strong and specific recognition abilities to the corresponding lectins, because of hydrophilicity. The interactions of glycopolymer layers with lectins were more specific and stronger than those of the saccharide monolayer, because the glycopolymer formed a thicker uniform hydrophilic layer. The thicker hydrophilic polymer layer provides 'free water' ${ }^{23}$ at the interface to prevent nonspecific protein adsorption. ${ }^{24-28}$ However, a small amount of nonspecific protein adsorption was still observed on the glycopolymer layer. Glycopolymers have multiple $\mathrm{OH}$ groups, which could disturb the water structure by hydrogen bonding and induce nonspecific adsorption. ${ }^{29}$ The hydrogen-bond formation of glycopolymers with proteins also induces nonspecific adsorption. In the case of the sugar monolayer, the surface was more hydrophobic, with a contact angle of $37^{\circ}$, and not only the hydrogen bonding of sugar but also hydrophobic interactions contributed to nonspecific adsorption.

Overall, glycopolymers exhibit strong affinity on the basis of multivalency and allosteric effects. ${ }^{30}$ On the whole, the glycopolymersubstituted substrate showed suitable properties for biosensing applications. Biosensing with a glycopolymer thin layer will be investigated in a later section.
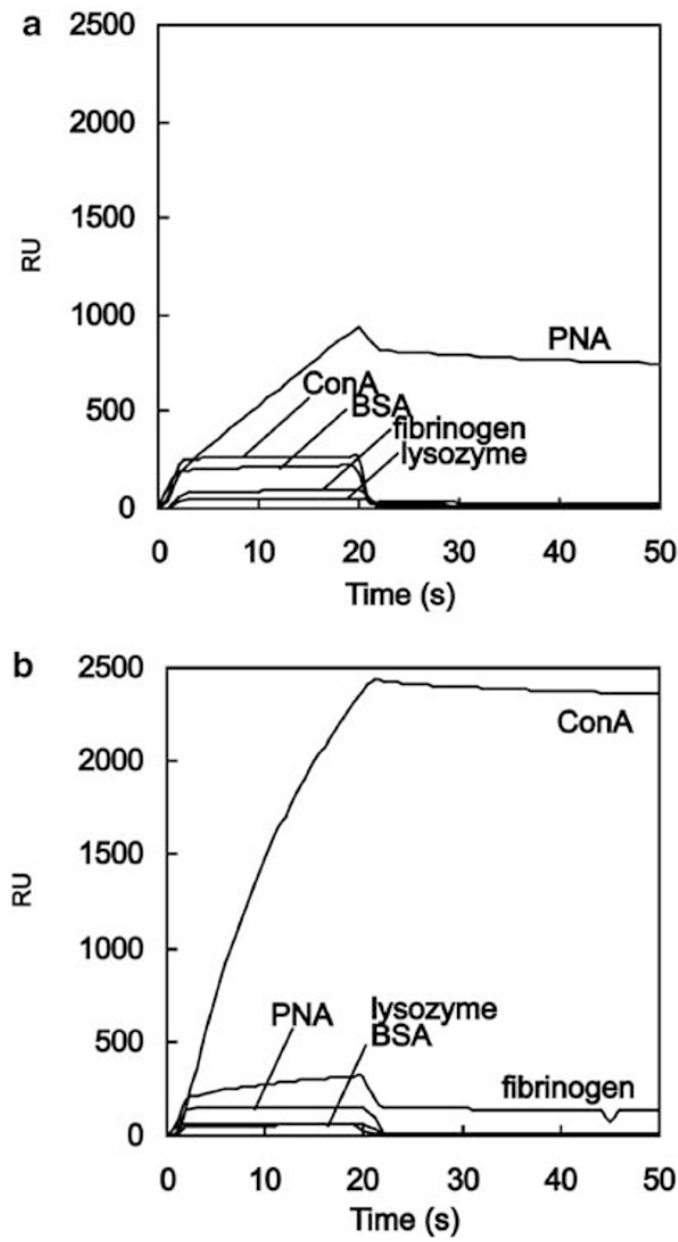

Figure 2 Surface plasmon resonance analyses of (a) P3-gold and (b) P6-gold with various proteins ( $500 \mathrm{~nm}$ ) in phosphate-buffered saline buffer at $25^{\circ} \mathrm{C}$.

Glycopolymer-substituted gold nanoparticles and lectin recognition Gold nanoparticles with glycopolymer substitution were prepared using the same polymers of $\mathbf{P 3}$ and $\mathbf{P 6}$, which are abbreviated as P3-GNP and P6-GNP, respectively. Nanoparticles were stably dispersed in an aqueous solution, and P3-GNP and P6-GNP showed a red color with $\lambda_{\max }$ at 529 and $524 \mathrm{~nm}$, respectively. Bonding of the glycopolymer on GNPs was calculated by weight change, comparing the weight measured before and after preparation. As a result, the area per molecule was $1.7 \mathrm{~nm}^{2}$ (Supplementary information). This result is consistent with that of gold substrates. Addition of the corresponding lectin ( $\mathrm{Gal}$ to PNA and Man to ConA) induced aggregation, resulting in a change in the color of the dispersion. In the case of P3-GNP, the addition of PNA induced aggregation and a red shift in $\lambda_{\max }$ from 529 to $531 \mathrm{~nm}$ (Figure $4 \mathrm{a}$ ). In contrast, $\lambda_{\max }$ did not shift with the addition of the non-corresponding proteins wheat germ agglutinin and BSA. Aggregation was confirmed by transmission electron microscopy observation. P3-GNP was placed on a grid, and the addition of PNA induced the formation of a $220 \times 280 \mathrm{~nm}^{2}$ aggregate of about 40 nanoparticles (Figures $4 \mathrm{~b}$ and c). Aggregates were not observed by UV-vis observation with the addition of ConA or BSA. In the case of P6-GNP, aggregation of nanoparticles and color changes were also observed with the addition of the corresponding lectin. 
Table 2 Kinetic constants for lectins with glycopolymer-modified substrate

\begin{tabular}{lccccc}
\hline Lectin & Substrate & $\mathrm{k}_{a}(1 / M s)$ & $\mathrm{k}_{d}(1 / \mathrm{s})$ & $\mathrm{K}_{A}(1 / M)$ & $R U_{\max }$ \\
\hline PNA & P3-Gold & $2.13 \times 10^{3}$ & $1.09 \times 10^{-4}$ & $1.99 \times 10^{7}$ & $9.43 \times 10^{2}$ \\
ConA & P3-Gold & - & - & - & $1.77 \times 10^{2}$ \\
PNA & P6-Gold & - & - & - & $5.07 \times 10^{2}$ \\
ConA & P6-Gold & $2.50 \times 10^{4}$ & $2.13 \times 10^{-3}$ & $1.64 \times 10^{7}$ & $3.10 \times 10$ \\
\hline
\end{tabular}

Abbreviations: ConA, concanavalin A; PNA, peanut agglutinin.

Table 3 SPR response of glycopolymer-modified substrate with proteins

\begin{tabular}{llll}
\hline & Substrate & $R U_{\max }$ & $R U_{\text {eq }}$ \\
\hline PNA & P3-Gold & $9.43 \times 10^{2}$ & $8.07 \times 10^{2}$ \\
ConA & P3-Gold & $1.77 \times 10^{2}$ & $3.10 \times 10$ \\
BSA & P3-Gold & $2.65 \times 10^{2}$ & $1.20 \times 10$ \\
Lysozyme & P3-Gold & $4.80 \times 10$ & 1.20 \\
Fibrinogen & P3-Gold & $9.70 \times 10$ & $1.90 \times 10$ \\
PNA & P6-Gold & $5.90 \times 10$ & $1.10 \times 10$ \\
ConA & P6-Gold & $2.38 \times 10^{3}$ & $2.41 \times 10^{3}$ \\
BSA & P6-Gold & $3.00 \times 10$ & 7.00 \\
Lysozyme & P6-Gold & $1.53 \times 10^{2}$ & 9.00 \\
Fibrinogen & P6-Gold & $3.17 \times 10^{2}$ & $1.46 \times 10^{2}$ \\
\hline
\end{tabular}

Abbreviations: BSA, bovine serum albumin; ConA, concanavalin A; PNA, peanut agglutinin.

\section{SPR signal amplification with glycopolymer-modified gold} nanoparticles

Surface plasmon resonance signal amplification was investigated with P3-GNP on the basis of local SPR. ${ }^{31,32}$ Figure 5 shows an SPR profile for a diluted protein concentration on an $\alpha$-Gal monolayer. The protein was first injected at a diluted concentration of $10 \mathrm{~nm}$, and the $\mathrm{RU}_{\mathrm{eq}}$ was difficult to observe because of the small amount of adsorption of the protein. The SPR response returned with the injection of P3-GNP. The SPR signal was not observed without the injection of proteins in advance. By contrast, this signal amplification was not observed with the P3-gold substrate.

The glycopolymer induced multiple binding to the sugar recognition sites, and PNA on P3-gold attached to P3-GNP, which induced an local SPR signal between P3-gold and P3-GNP. The binding of the $\alpha$-Gal monolayer to proteins was nonspecific (Figure $3 b$ ).

a

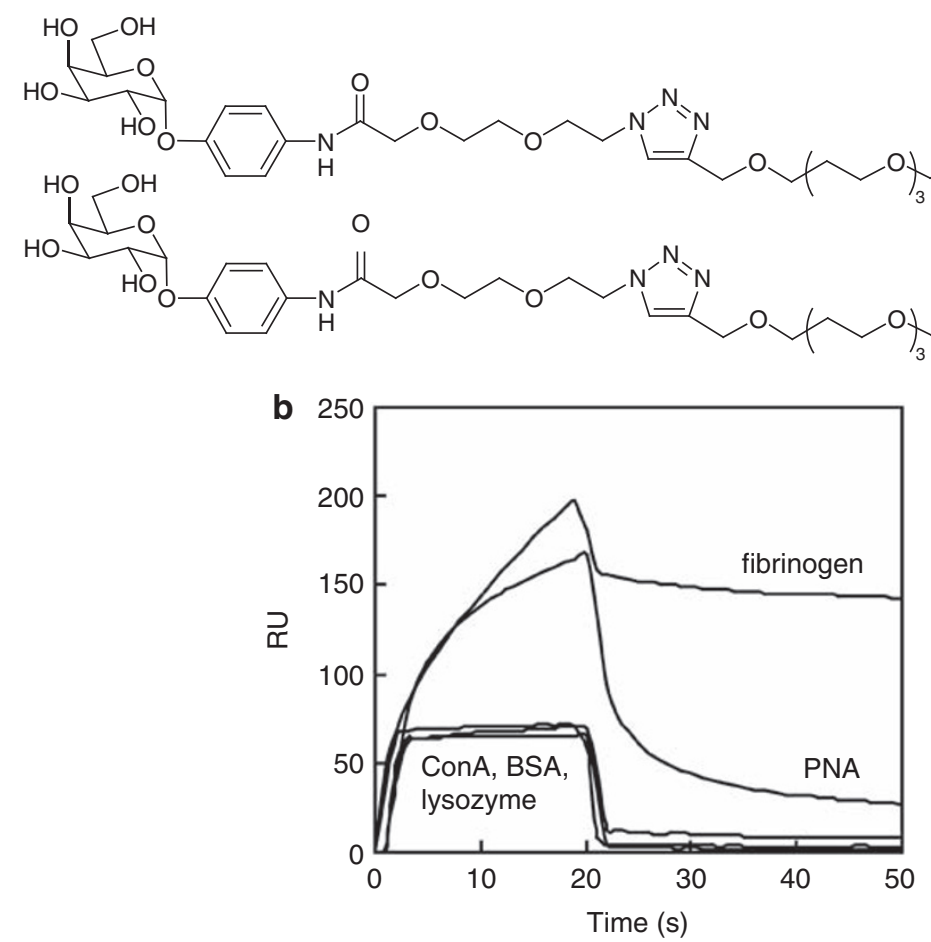

Figure 3 Binding analyses of $\alpha$-Gal monolayers. (a) A schematic illustration of $\alpha$-Gal on the gold substrate. (b) Surface plasmon resonance analyses of $\alpha$-Gal monolayers with various proteins ( $500 \mathrm{~nm}$ ) in phosphate-buffered saline buffer at $25^{\circ} \mathrm{C}$. 


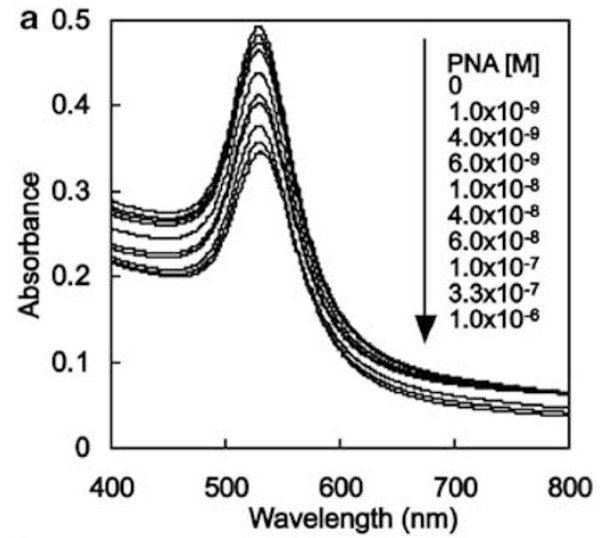

b

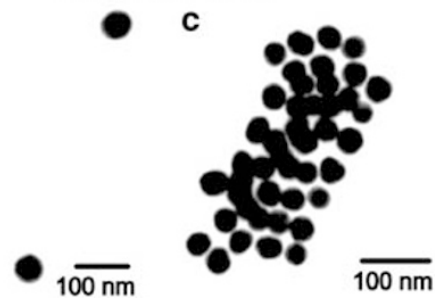

Figure 4 Observations of P3-GNP with the addition of proteins. (a) Ultravisible spectra of P3-GNP with the addition of peanut agglutinin. Transmission electron microscopy observations (b) without the addition of proteins and (c) with the addition of peanut agglutinin

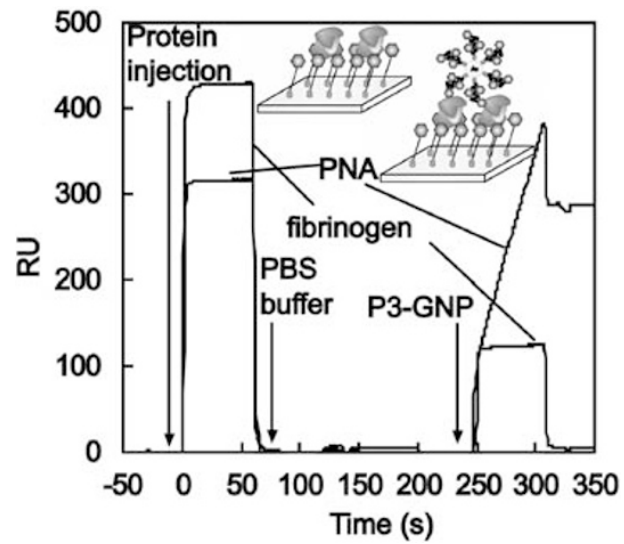

Figure 5 Signal amplification of surface plasmon resonance by the addition of P3-GNP $\left(\mathrm{OD}_{529}=0.45\right)$ and a schematic illustration for a protein concentration of $10 \mathrm{~nm}$.

The injection of glycopolymer-substituted GNPs can be used to visualize the specific binding of PNA because of the binding specificity of P3, but the signal amplification can be observed only for a slow flow rate $\left(10 \mu \mathrm{lmin}^{-1}\right)$ because of the weak interaction. The combination of a saccharide-modified substrate and nanoparticles enables the sensitive detection of proteins.

\section{Biosensing of shiga toxin-1 using glycopolymer-modified} substances

We investigated a more practical target and biosensing using glycopolymer-substituted materials. Considering that $\alpha$-Gal-terminated saccharides bind to Stxs, we studied the interaction of Stx-1 with P3-gold and P3-GNP.

The P3-gold substrate was subject to measurements by SPR for determining the interaction with $\mathrm{Stx}-1 . \mathrm{RU}_{\max }$ showed a clear dose
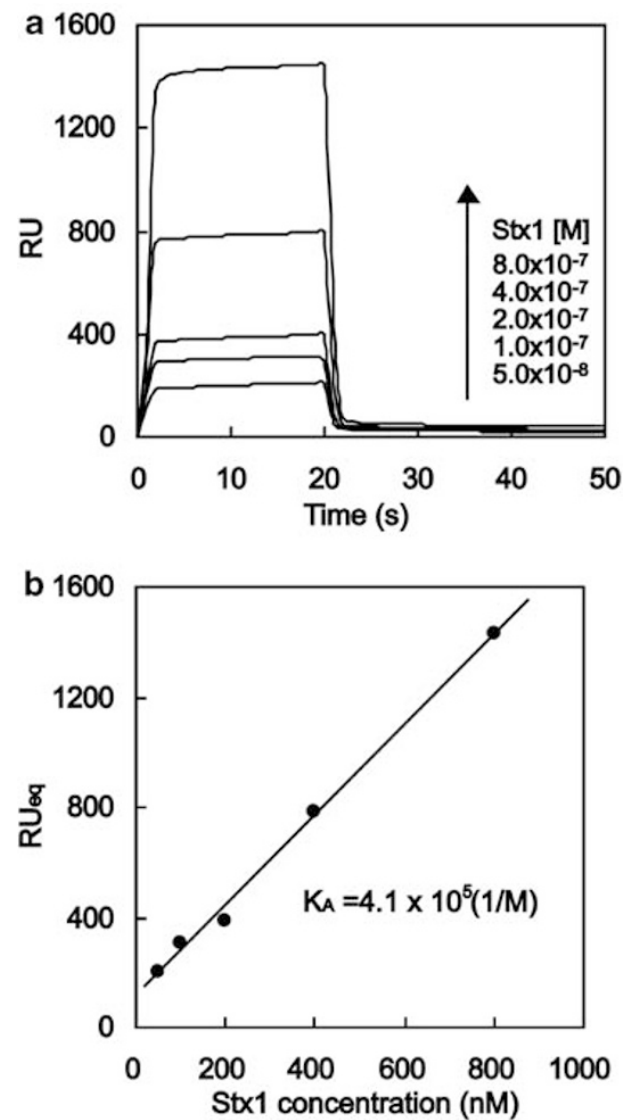

Figure 6 (a) Surface plasmon resonance analyses of P3-gold with Stx-1 and (b) Scatchard plot of P3-gold with Stx-1.

dependency on the concentration of Stx-1, and the binding constant based on the Scatchard analysis was $4.1 \times 10^{5} \mathrm{M}^{-1}$ (Figure 6). P3-GNP showed a slight increase in ultraviolet absorbance with the addition of Stx-1, and P3-GNP formed small aggregates in the solution (Figure 7). Finally, the immunochromatography of Stx-1 was investigated. A vague pink spot was observed with the addition of $2.4 \mu \mathrm{M}$ Stx-1, although the spot was not observed with other proteins (Supplementary information).

The affinity of P3-gold to Stx-1 was not sufficient for detecting Stx- 1 because the pendant saccharide structure of $\alpha$-Gal is different from the natural ligand structure of Gb3 (Gal $\alpha 1-4 \mathrm{Gal} \beta 1-4 \mathrm{GlcCer}){ }^{33}$ However, these results indicate that the glycopolymer thin layer can be applied for the biosensing of sugar recognition toxins. The immunochromatography of glycopolymer-substituted nanoparticles is still under investigation with glycopolymers carrying a natural ligand, in order to develop highly sensitive immunochromatography.

\section{CONCLUSION}

Gold surfaces modified with glycopolymers were prepared by living radical polymerization with RAFT. We prepared glycopolymers using a range of saccharides, molecular weights and sugar densities. Thiolterminated glycopolymers were used for the modification of gold substrates and GNPs. The glycopolymer-substituted gold substrates showed strong, specific molecular recognition abilities with lectins, because of the hydrophilic polymer properties. Biosensing using glycopolymer-modified GNPs showed specific interactions with target proteins and toxins. 

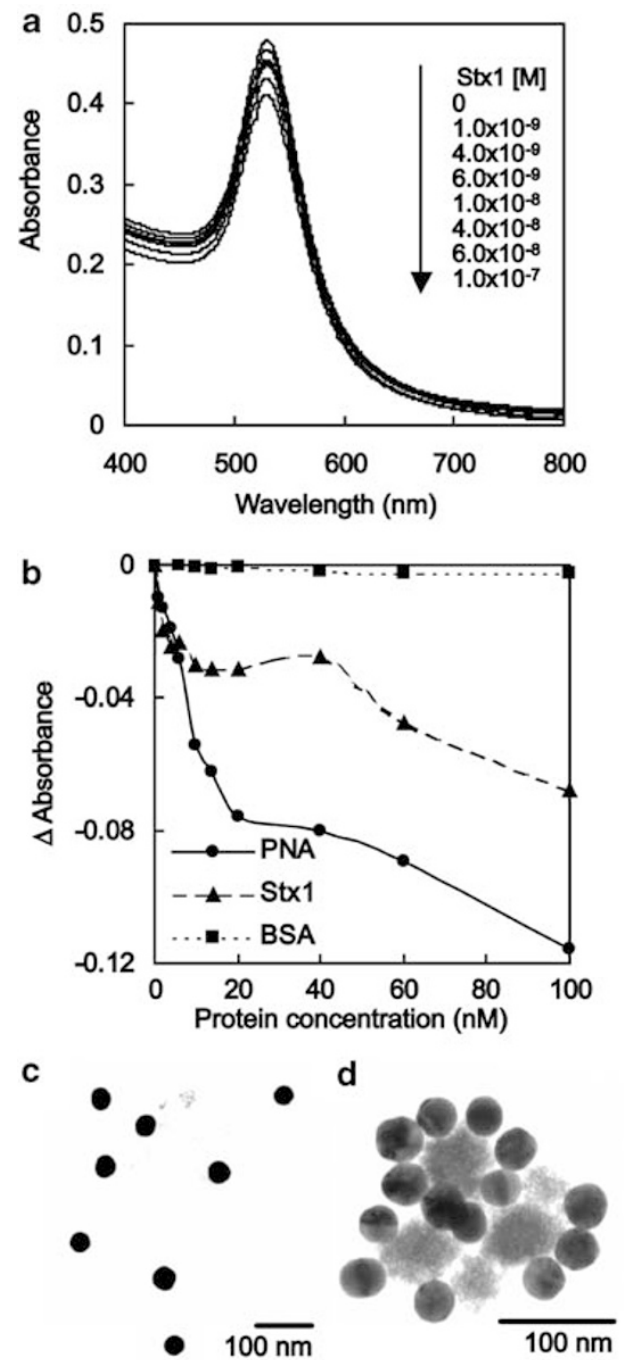

Figure 7 Ultra-visible spectra of (a) P3-GNP with the addition of Stx1 and (b) the intensity at $529 \mathrm{~nm}$ for varying protein concentrations $((\bullet)$ peanut agglutinin, ( $\mathbf{\Lambda})$ Stx1 and ( $\boldsymbol{\square})$ bovine serum albumin). Transmission electron microscopy micrograph of Stx-1 stained with 1 wt\% $\mathrm{OsO}_{4}$ with P3-GNP. The entire image of Stx-1 (c) before and (d) after the addition of Stx1.

\section{Electronic supporting information available}

The syntheses of glycomonomers, atomic force microscopy imaging of the glycopolymer-modified layer, SPR analyses, evaluation of GNPs and immunochromatography are available through the Internet.

\section{ACKNOWLEDGEMENTS}

This work was supported by a Grant-in-Aid for Young Scientists (B) (20750088).

1 Taylor, M. E. \& Drickamaer, K. Introduction to Glycobiology (Oxford Press, London, 2002).

2 Jelinek, R. \& Kolusheva, S. Carbohydrate biosensors. Chem. Rev. 104, 5987-6016 (2004).

3 Mammen, M., Choi, S. K. \& Whitesides, G. M. Polyvalent interactions in biological systems: implications for design and use of multivalent ligands and inhibitors. Angew. Chem. Int. Ed. 37, 2754-2794 (1998).

4 Lee, T. C. \& Lee, R. T. Carbohydrate-protein interactions: basis of glycobiology. Acc. Chem. Res. 28, 321-327 (1995).
5 Taylor, M. E., Bezouska, K. \& Drickamer, K. Contribution to ligand binding by multiple carbohydrate-recognition domains in the macrophage mannose receptor. J. Biol. Chem. 267, 1719-1726 (1992).

6 Miura, Y., Ikeda, T. \& Kobayashi, K. Chemoenzymatically synthesized glycoconjugate polymers. Biomacromolecules 4, 410-415 (2003).

7 Miura, Y., Sasao, Y., Dohi, H., Nishida, Y. \& Kobayashi, K. Self-assembled monolayers of globotriaosylceramide (Gb3) mimics: surface specific affinity with shiga toxins. Anal. Biochem. 310, 27-35 (2002).

8 Miura, Y., Yasuda, K., Yamamoto, K., Koike, M., Nishida, Y. \& Kobayashi, K. Inhibition of alzheimer amyloid aggregation with sulfated glycopolymers. Biomacromolecules $\mathbf{8}$, 2129-2134 (2007).

9 Toyoshima, M. \& Miura, Y. Preparation of glycopolymer-substituted gold nanoparticles and their molecular recognition. J. Polym. Sci. Part A: Polym. Chem. 47, 1412-1421 (2009).

10 Moad, G., Rizzardo, E. \& Tang, S. H. Living radical polymerization by the RAFT process. Aus. J. Chem. 58, 379-410 (2005).

11 Housni, A., Cai, H., Liu, S., Pun, S.H. \& Narain, R. Facile preparation of glyconanoparticles and their bioconjugation to streptavidin. Langmuir 23, 5056-5061 (2007).

12 Kitano, H., Anraku, Y. \& Shinohara, H. Sensing capabilities of colloidal gold monolayer modified with a phenylboronic acid-carrying polymer brush. Biomacromolecules 7 , 1065-1071 (2006).

13 McCormick, C. L. \& Lowe, A. B. Aqueous RAFT polymerization: recent developments in synthesis of functional water-soluble (Co)polymers with controlled structures. Acc. Chem. Res. 37, 312-325 (2004).

14 Wageesha, S., Luisa, A. \& Christopher, K. O. Self-assembled monolayers and polymer brushes in biotechnology: current applications and future perspectives. Biomacromolecules 6, 2427-2448 (2005).

15 Uzawa, H., Ito, H., Neri, P., Mori, H. \& Nishida, Y. Glycochips from polyanionic glycopolymers as tools for detecting shiga toxins. Chem. Biochem. 8, 2117-2124 (2007).

16 Matsumoto, E., Yamauchi, T., Fukuda, T. \& Miura, Y. Sugar microarray via click chemistry: molecular recognition with lectins and amyloid $\beta$ (1-42). Sci. Technol. Adv. Mater. 10, 34605-34611 (2009).

17 Miura, Y., Yamauchi, T., Sato, H. \& Fukuda, T. The self-assembled monolayer of saccharide via click chemistry: Formation and protein recognition. Thin Solid Films 516, 2443-2449 (2008)

18 Levy, D. E. \& Fugedi, P. Organic Chemistry of Sugars (Marcel Dekker Inc., New York, USA, 2005).

19 Nagatani, N., Tanaka, R., Yuhi, T., Endo, T., Kerman, K., Takamura, Y. \& Tamiya, E. Gold nanoparticle-based novel enhancement method for the development of highly sensitive immunochromatographic test strips. Sci. Technol. Adv. Mater. 7, 270-275 (2006).

20 Thomas, D. B., Sumerlin, B. S., Lowe, A. B. \& McCormick, C. L. Conditions for facile, controlled RAFT polymerization of acrylamide in water. Macromolecules 36 , 1436-1439 (2003).

21 Sumerlin, B. S., Lowe, A. B., Stroud, P. A., Zhang, P., Urban, M. W. \& McCormick, C. L. Modification of gold surfaces with water-soluble (Co)polymers prepared via aqueous reversible additionfragmentation chain transfer (RAFT) polymerization. Langmuir 19, 5559-5562 (2003).

22 Chakrabarti, A. Monte Carlo study of pancake to brush transition. J. Chem. Phys. 100, 631-635 (1994).

23 Tanaka, M., Mochizuki, A., Ishii, N., Motomura, T. \& Hatakeyama, T. Study of blood compatibility with Poly(2-methoxyethyl acrylate). Relationship between water structure and platelet compatibility in poly(2-methoxyethylacrylate-co-2-hydroxyethylmethacrylate). Biomacromolecules 3, 36-41 (2002).

24 Ide, M., Mori, T., Ichikawa, K., Kitano, H., Tanaka, M., Mochizuki, A., Oshiyama, H. \& Mizuno, W. Structure of water sorbed into poly(MEA-co-HEMA) films as examined by ATR-IR spectroscopy. Lagnmuir 19, 429-435 (2003).

25 Ladam, G., Vonna, L. \& Sackmann, E. Micromechanics of surface-grafted hyaluronic acid gels. J. Phys. Chem. B. 107, 8965-8971 (2003).

26 Prime, K. L. \& Whitesides, G. M. Self-assembled organic monolayers: model systems for studying adsorption of proteins at surfaces. Science 252, 1164-1167 (1991).

27 Satomi, T., Nagasaki, Y., Kobayashi, H., Otsuka, H. \& Kataoka, K. Density control of Poly(ethylene glycol) layer to regulate cellular attachment. Langmuir 23, 6698-6703 (2007).

28 Waranabe, J. \& Ishihara, K. Cell engineering biointerface focusing on cytocompatibility using phospholipid polymer with an isomeric oligo(lactic acid) segment. Biomacromolecules 6, 1797-1802 (2005).

29 Kitano, H., Tada, S., Mori, T., Takaha, K., Gemmei-Ide, M., Tanaka, M., Fukuda, M. \& Yokoyama, Y. Correlation between the structure of water in the vicinity of carboxybetaine polymers and their blood-compatibility. Langmuir 21, 11932-11940 (2005).

30 Nagahori, N. \& Nishimura, S. -l. Tailored glycopolymers: controlling the carbohydrateprotein interaction based on template effect. Biomacromolecules 2, 22-23 (2001).

31 Kawaguchi, T., Shankaran, D. R., Kim, S. -J., Matsumoto, K., Toko, K. \& Miura, N. Surface plasmon resonance immunosensor using Au nanoparticle for detection of TNT. Sensors Actuators B Chem. B. 133, 467-472 (2008).

32 Wilets, K. A. \& Van Duyne, R. P. Localized surface plasmon resonance spectroscopy and sensing. Annu. Rev. Phys. Chem. 58, 267-297 (2007).

33 Lingwood, C. A., Law, H., Richardson, S., Petric, M., Brunton, J. L., Grandis, S. D. \& Karmali, M. Glycolipid binding of purified and recombinant Escherichia coli produced verotoxin in vitro. J. Biol. Chem. 262, 8834-8839 (1987).

Supplementary Information accompanies the paper on Polymer Journal website (http://www.nature.com/pj) 